\title{
On mechanical properties of planar flexure hinges of compliant mechanisms
}

\author{
F. Dirksen and R. Lammering \\ Institute of Mechanics, Helmut-Schmidt-University/University of the Federal Armed Forces Hamburg, \\ Holstenhofweg 85, 22043 Hamburg, Germany
}

Received: 25 February 2011 - Revised: 28 April 2011 - Accepted: 23 May 2011 - Published: 6 June 2011

\begin{abstract}
The synthesis of compliant mechanisms yield optimized topologies that combine several stiff parts with highly elastic flexure hinges. The hinges are often represented in finite element analysis by a single node (one-node hinge) leaving doubts on the physical meaning as well as an uncertainty in the manufacturing process.
\end{abstract}

To overcome this one-node hinge problem of optimized compliant mechanisms' topologies, one-node hinges need to be replaced by real flexure hinges providing desired deflection range and the ability to bear internal loads without failure. Therefore, several common types of planar flexure hinges with different geometries are characterized and categorized in this work providing a comprehensive guide with explicit analytical expressions to replace one-node hinges effectively.

Analytical expressions on displacements, stresses, maximum elastic deformations, bending stiffness, center of rotation and first natural frequencies are derived in this work. Numerical simulations and experimental studies are performed validating the analytical results. More importance is given to practice-oriented flexure hinge types in terms of cost-saving manufacturability, i.e. circular notch type hinges and rectangular leaf type hinges.

\section{Introduction}

In order to create machine tools for small scale applications, compliant mechanisms $(\mathrm{CM})$ have become more popular in the last years competing against rigid body systems connected by conventional pin joints. CM are flexible, monolithic structures that gain their motion from the (elastic) deformation of certain parts, so-called flexure hinges. CM are potentially more accurate, better scalable, cleaner, less noisy and most importantly more cost-saving in manufacturing and maintenance. However, designing CM is more difficult and non-intuitive due to its inherent complex overall deformation.

Several approaches have arisen to address this drawback by applying numerical topology design and optimization procedures. Relevant contributions have been made by various research teams, in particular, Ananthasuresh and Kota (1995), Frecker et al. (1997),

Correspondence to: F. Dirksen (frank.dirksen@hsu-hh.de)
Saxena and Ananthasuresh (2000), Howell (2001), Bruns and Tortorelli (2001), Ansola et al. (2002), Bendsøe and Sigmund (2003), Mattson et al. (2004), Bendsøe and Sigmund (2008). All these techniques lead in a systematic manner to final, optimized topologies, i.e. an optimal distribution of material over the design domain is obtained to meet the userspecified motion requirements. As a key result, one-node hinges (often called pseudo-hinges) with doubtful physical meaning arise. As an example, a gripping mechanism and a close up of a one-node hinge, obtained by a topology optimization procedure without any regularization, is shown in the upper box in Fig. 1. Although some techniques exist circumventing this critical issue, e.g. Poulsen (2002), Yoon et al. (2004) or Sigmund (2009), a more consequent way is to use the already known data from the finite element calculation used in the topology optimization process. Since nodal displacements for a given topology are known, the required deflection range and (internal) nodal forces are available without additional costs, as well. These information can be used to replace one-node hinges with real flexure hinges that meet the deflection and load bearing requirements as a result of their specific shape, dimension and material data. 


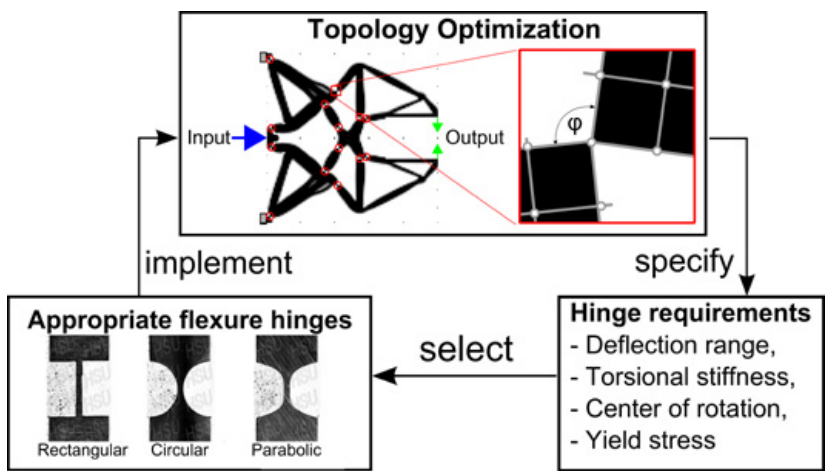

Figure 1. Beneficial procedure for non-intuitive synthesis of compliant mechanisms: Replacing artificial one-node hinges by appropriate flexure hinge types meeting specified, known hinge requirements.

Necessary mechanical properties of flexure hinges have been investigated by a few authors. Paros and Weisbord (1965) did pioneer work yielding approximate compliances of flexure hinges decades ago. Smith (2000) provided in his book a good background on flexure elements and some flexure systems. Lobontiu (2003) analytically investigated flexure hinges based on energy principles to calculate desired properties at individual single points of hinges. Recently, Raatz (2006) demonstrated in her dissertation the potential of flexure hinges in compliant parallel mechanisms using superelastic shape memory alloys.

In spite of the aforementioned research, the mechanical behavior of flexure hinges is not yet fully characterized in terms of the synthesis of compliant mechanisms and, thus, leaving a gap between final, optimized topologies and appropriate flexure hinges. In order to bridge this gap, mechanical properties of flexure hinges are derived and validated in this work to provide a comprehensive guide from a topology optimization standpoint. The overall scheme is shown in Fig. 1.

\section{Objectives}

For the synthesis of compliant mechanisms it is crucial to characterize and categorize individual flexure hinges in terms of their mechanical properties as a result of geometric shape and material data. Therefore, relevant mechanical properties are derived, such as:

- Displacements $u(x, z), w(x)$, to gain a better understanding of the deformation of the whole flexure hinge.

- Mechanical stresses $\sigma_{x}(x, z), \tau_{x z}(x, z)$, to identify critical regions that are not apparent.

- Stiffness $k_{z}$ and bending stiffness $c_{\psi}$, to be able to model flexure hinges appropriately by spring joints.

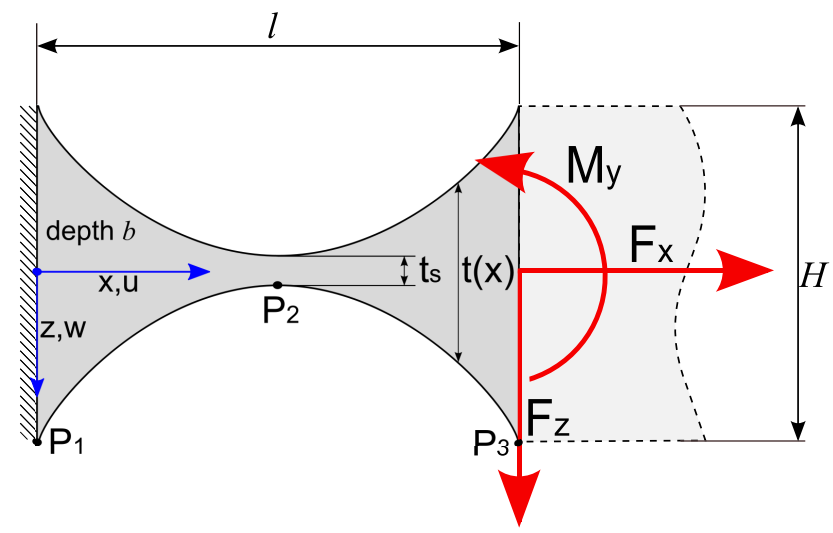

Figure 2. Planar, flexure hinge characterized by length $l$, depth $b$, height $H$, variable thickness $t(x) \geq t_{s}$ and common points $P_{1}, P_{2}, P_{3}$ to resist external (nodal) loads $F_{x}, F_{z}, M_{y}$.

- Center of rotation and its motion with deflection, to identify and compensate a change of kinematics under certain loading conditions.

- Maximum (elastic) deformation, to identify deflection limits and avoid material failure.

- Natural frequencies $f$, to understand the behavior under dynamic load conditions and to check the quality of numerical simulations against experimental data.

Analytical expressions are derived using a standard x-zcoordinate system, as shown in Fig. 2, by applying different established theories and models. If possible, numerical simulations and experimental data are used to validate the analytical calculations.

In this work, planar flexure hinges of different geometries are examined: rectangular, circular and parabolic flexure hinges, denoted by superscripts $\mathrm{R}, \mathrm{C}, \mathrm{P}$ respectively, are used due to an easy manufacturability $(\mathrm{R}, \mathrm{C})$ and convenient mathematical handling $(\mathrm{R}, \mathrm{P})$. In particular, circular shape is approximated by parabolic function using Taylor expansion to avoid complicated expressions. The geometric approximation error was checked and is negligible in all loaded regions.

The geometry of flexure hinges is described by length $l$, height $H$ and variable thickness $t(x) \geq t_{s}$ as well as common points $P_{1}\left(0, \frac{H}{2}\right), P_{2}\left(\frac{l}{2}, \frac{t_{s}}{2}\right)$ and $P_{3}\left(l, \frac{H}{2}\right)$, as shown in Fig. 2. The depth is set to uniform $b=10 \mathrm{~mm}$ over the entire hinge, which is sufficient for the majority of planar applications.

Key aspect for the following calculations is the geometric shape given by the variable thickness $t(x)$ of each type of flexure hinge

$t^{\mathrm{R}}(x)=t_{s}$, 


$$
\begin{aligned}
t^{\mathrm{P}}(x) & =2\left(c_{1}+c_{2} x+c_{3} x^{2}\right) \\
& =H-\frac{4 x\left(H-t_{s}\right)}{l}+\frac{4 x^{2}\left(H-t_{s}\right)}{l^{2}}, \\
t^{\mathrm{C}}(x) & =2\left(z_{M}+\sqrt{r^{2}-\left(x-x_{M}\right)^{2}}\right) \\
& =\frac{H^{2}-t_{s}^{2}+l^{2}}{4\left(H-t_{s}\right)}-\sqrt{\frac{\left(l^{2}+\left(H-t_{s}\right)^{2}\right)^{2}}{\left(4\left(H-t_{s}\right)\right)^{2}}-\frac{(l-2 x)^{2}}{4}} .
\end{aligned}
$$

Parabolic and circular hinges are first written in a general form denoted by polynomial coefficients $c_{1}, c_{2}, c_{3}$ and circle's center coordinates $x_{M}, z_{M}$ and radius $r$, respectively. In the second lines of Eqs. (2) and (3), relevant geometric boundary conditions

$$
\begin{aligned}
& c_{1}=\frac{H}{2}, \quad c_{2}=\frac{-2\left(H-t_{s}\right)}{l}, \quad c_{3}=\frac{2\left(H-t_{s}\right)}{l^{2}}, \\
& x_{M}=\frac{l}{2}, \quad z_{M}=\frac{t_{s}}{2}+r, \quad r=\frac{l^{2}+\left(H-t_{s}\right)^{2}}{4\left(H-t_{s}\right)},
\end{aligned}
$$

are applied. Throughout this paper, the formulations $t^{\mathrm{R}, \mathrm{P}, \mathrm{C}}=t^{\mathrm{R}, \mathrm{P}, \mathrm{C}}\left(x, H, l, t_{s}\right)$ are used to keep the solution adaptable to specific problems.

In order to compare analytical results with numerical and experimental data, a high strength aluminum wrought alloy $\mathrm{AlCu}_{4} \mathrm{Mg}_{1}$ (EN AW 2024) that is often used in applications of $\mathrm{CM}$ due to its high fatigue strength and high elastic strain, is considered throughout this work. The relevant material specifications are

$E=70 \mathrm{GPa}, \quad v=0.33, \quad \rho=2790 \mathrm{~kg} \mathrm{~m}^{-3}$.

Although it remains unchanged throughout this publication, the analytical formulas hold for other isotropic materials as well.

\section{Mechanical properties of flexure hinges}

Relevant mechanical properties of individual flexure hinges under quasi-static loading conditions are described and discussed in this section. The total behavior of CM consisting of several flexure hinges is not described here and is subject to further investigations. Since flexure hinges are mainly used in $\mathrm{CM}$ to allow rotational motion, the main focus is on axial bending caused by external nodal forces $F_{x}, F_{z}$ and moment $M_{y}(x)$ as illustrated in Fig. 2.

\subsection{Moments of area}

The areas of the cross section $A(x)=b t_{s}(x)$, first moments of area $S_{y}(x, z)$ and second moments of area $I_{y}(x)$ were calculated and are listed in Table 1 for all considered flexure hinges using thicknesses $t(x)$ given in Eqs. (1)-(3). Note, that the first moment of area is calculated from $z$ to $t(x) / 2$.

The listed moments of area are used to calculate stresses and displacements in the following sections.
Table 1. First and second moments of areas of rectangular (R), circular $(\mathrm{C})$ and parabolic $(\mathrm{P})$ flexure hinges.

\begin{tabular}{ccc}
\hline & $S_{y}(x, z)=\int_{A^{*}} z^{*} d A$ & $I_{y}(x)=\int_{A} z^{2} d A$ \\
\hline $\mathrm{R}$ & $\frac{b}{8}\left(t_{s}^{2}-z^{2}\right)$ & $\frac{b t_{s}^{3}}{12}$ \\
$\mathrm{C}$ & $\frac{b\left(\left(z_{M}+\sqrt{r^{2}-\left(x-x_{M}\right)^{2}}\right)^{2}-z^{2}\right)}{2}$ & $\frac{b\left(\left(x-x_{M}\right)^{2}+2 r\left(z_{M}-r\right)\right)^{3}}{12 r^{3}}$ \\
$\mathrm{P}$ & $\frac{b\left(\left(H l^{2}+4\left(H-t_{s}\right)\left(x^{2}-l x\right)\right)^{2}-4 l^{4} z^{2}\right)}{8 l^{4}}$ & $\frac{b\left(H(l-2 x)^{2}+4 t_{s}(l-x) x\right)^{3}}{12 l^{6}}$ \\
\hline
\end{tabular}

\subsection{Stresses}

The normal stresses $\sigma_{x}(x, z)=\frac{F_{x}}{A(x)}+\frac{M_{y}(x)}{I_{y}(x)} z$, and shear stresses $\tau_{x z}=\frac{F_{z}(x) S_{y}(x, z)}{I_{y}(x) b}$ depend on the external loads, moments of area $S_{y}(x, z), I_{y}(x, z)$ and depth $b$, where a linear-elastic, isotropic stress-strain relation is assumed. Furthermore, the normal stresses $\sigma_{y}, \sigma_{z}$ and shear stresses $\tau_{y z}, \tau_{x y}$ are assumed to be negligible. Thus, the relevant normal stresses are

$$
\begin{aligned}
\sigma_{x}^{\mathrm{R}}(z) & =\frac{1}{b t_{s}} F_{x}+\frac{12(x-l) z}{b t_{s}^{3}} F_{z}+\frac{12 z}{b t_{s}^{3}} M_{y}, \\
\sigma_{x}^{\mathrm{P}}(x, z) & =\frac{l^{2}}{b h_{*}^{3}(x)} F_{x}+\frac{12 l^{6} z}{b h_{*}^{9}(x)} M_{y}+\frac{12 l^{6}(x-l) z}{b h_{*}^{9}(x)} F_{z}, \\
\sigma_{x}^{\mathrm{C}}(x, z) & =\frac{1}{2 b h_{* *}(x)} F_{x}+\frac{3 z(x-l)}{2 b h_{* *}(x)} M_{y}+\frac{3 z}{2 b h_{* *}(x)} F_{z},
\end{aligned}
$$

and shear stresses are

$$
\begin{aligned}
\tau_{x z}^{\mathrm{R}}(z) & =\left(\frac{-6 z^{2}}{b t_{s}^{3}}+\frac{3}{2 b t_{s}}\right) F_{z}, \\
\tau_{x z}^{\mathrm{P}}(x, z) & =\left(\frac{3 l^{2}\left(-4 l^{4} z^{2}+h_{*}^{6}(x)\right)}{2 b h_{*}^{9}(x)}\right) F_{z}, \\
\tau_{x z}^{\mathrm{C}}(x, z) & =\frac{3\left(r^{2}-z^{2}-\left(x-x_{M}\right)^{2}+\left(z_{M}-2 \sqrt{\left.r^{2}-\left(x-x_{M}\right)^{2}\right)}\right) z_{M}\right)}{4 b h_{* *}(x)} F_{z},
\end{aligned}
$$

where $h_{*}^{3}(x)=H(l-2 x)^{2}+4(l-x) x t_{s}$ and $h_{* *}(x)=z_{M}-$ $\sqrt{r^{2}-\left(x-x_{M}\right)^{2}}$ are introduced to keep the expressions short. Note, that any stress concentration effects are not yet taken into account as they will later, in Sect. 3.4.

\subsection{Displacements}

The displacements $u(x, z), w(x)$ due to external loads $F_{x}, F_{z}$, $M_{y}$, as shown in Fig. 2, are calculated. Later, they are used to calculate stiffness and bending stiffness in Sect. 3.5.

In order to calculate displacements $u(x, z), w(x)$ and bending slope $\psi(x)$, different beam theories are supposed to be applicable: Euler-Bernoulli's beam theory assumes that the (shear-indeformable) cross section remains perpendicular to the neutral axis and $\psi \approx \tan \psi=-w^{\prime}(x)$, which is sufficient 
for slender beams (e.g. rectangular flexure hinges) undergoing small and moderate bending angles. Elastica beam theory lifts the latter limitation using the correct, non-linear expression $\frac{w_{E}^{\prime \prime}(x)}{\left(1+\left(w_{E}^{\prime}(x)\right)^{2}\right)^{3 / 2}}=-\frac{M_{y}(x)}{E I_{y}(x)}$ and, thus, it also holds for large bending angles. Timoshenko's beam theory holds for small and moderate bending angles, as Bernoulli's theory does, but it takes the shear deformation caused by arising shear stresses into account. Usually, this has a minor effect on the displacements considering "long" rectangular hinges $(t(x) \ll l)$. However, it cannot be neglected in the case of "thick" hinges with an increased "effective" thickness $\left(t^{\mathrm{eff}}=\frac{1}{l} \int t(x) d x \approx l\right)$ compared to hinge length $l$, such as most circular and parabolic hinges. Further details can be found in standard literature; particularly the influence of large deformations and shear stresses are described in Love (1920) and Wang et al. (2000), respectively.

In this work, Timoshenko's beam theory is used to calculate the required displacements, since flexure hinges do not undergo large rotations and shear deformation cannot be neglected. The displacement expressions are

$$
\begin{aligned}
& w^{\prime}(x)=-\psi(x)+\frac{F_{z}}{\alpha_{S} G A(x)}, \\
& \psi^{\prime}(x)=\frac{M_{y}(x)}{E I_{y}(x)} \\
& u(x, z)=z \psi(x)+\int_{0}^{x} \frac{F_{x}}{E A\left(x^{*}\right)} d x^{*} .
\end{aligned}
$$

Here, the angles $w^{\prime}(x)$ and $\psi(x)$ differ by an additional shear deformation term, where $\alpha_{s}$ is a shear correction factor compensating non-uniform shear stresses $\tau_{x s}$ in the cross section. Furthermore, the displacement $u(x, z)$ is expanded by an additional axial displacement term caused by axial forces $F_{x}$.

Based on Eq. (7), the displacement expressions can be calculated for different types of flexure hinges. As an example, the displacements for a rectangular flexure hinge based on Timoshenko's theory become

$$
\begin{aligned}
w^{\mathrm{R}}(x) & =\frac{12(1+v) t_{s}^{2} x+30 l x^{2}-10 x^{3}}{5 E b t_{s}^{3}} F_{z}-\frac{6 x^{2}}{E b t_{s}^{3}} M_{y}, \\
\psi^{\mathrm{R}}(x) & =-\frac{12 l x-6 x^{2}}{E b t_{s}^{3}} F_{z}+\frac{12 x}{E b t_{s}^{3}} M_{y}, \\
u^{\mathrm{R}}(x, z) & =\frac{x}{E b t_{s}} F_{x}-\frac{\left(12 l x-6 x^{2}\right) z}{E b t_{s}^{3}} F_{z}+\frac{12 x z}{E b t_{s}^{3}} M_{y} .
\end{aligned}
$$

The derived displacements expressions are used in Sect. 3.5 to calculate stiffness and bending stiffness of different flexure hinges. Note, that anti-clastic bending effects are neglected, as suggested by Conway and Nickola (1965).

\subsection{Maximum elastic deformation}

Flexure hinges can undergo smaller rotational deformation than conventional pin joints that have practically no limits. The maximum elastic deformation of flexure hinges can be estimated by combining the occurring stresses derived above to an equivalent stress $\sigma_{V}$ which has to be lower than the yield stress $\mathrm{R}_{p 0.2}: \sigma_{V} \leq \mathrm{R}_{p 0.2}$. Among various established yield criteria, von-Mises yield criterion $\sigma_{V}=\sqrt{\sigma_{x}^{2}+3 \tau_{x z}^{2}}$ is mainly used for ductile materials and, thus, applicable to the majority of materials in compliant mechanisms.

Static load cases and quasi-static motions are considered; fatigue effects and durability are not yet fully investigated and will be subject of future investigations and publications.

Maximum normal stress $\sigma_{x, \max }$ can be found at the thinnest cross section $x=x\left(t=t_{s}\right)$ at the upper or lower edge $z= \pm t_{s} / 2$. Whereas maximum shear stresses $\tau_{x z \text { max }}$ occur at the center of the thinnest cross section $x=x\left(t=t_{s}\right)$ at $z=0$ and is zero at the edges $\tau_{x z}\left(z= \pm t_{s} / 2\right)=0$. Typically in applications considered here, normal stresses are more dominant than shear stresses suggesting to neglect shear stress. However, maximum shear stresses are taken into account in the equivalent stress due to safety reasons in this work. Therefore, equivalent stresses become

$$
\begin{aligned}
\sigma_{V, \max } & =\sqrt{\left(\sigma_{\max , x} K_{t x}+\sigma_{\max , b} K_{t b}\right)^{2}+3 \tau_{\max }^{2}} \\
& =\sqrt{\left(\frac{F_{x}}{b t_{s}} K_{t x}+\frac{6 M_{y}(x)}{b t_{s}^{2}} K_{t b}\right)^{2}+3\left(\frac{F_{z}}{2 b t_{s}}\right)^{2}},
\end{aligned}
$$

where stress concentration factors $K_{t x}$ and $K_{t b}$ for axial and bending loads (second indices $x, b$ ) are introduced.

For rectangular leaf type hinges, with uniform thickness $t(x)=t_{s}=$ const. , the critical section is solely determined by the maximum bending moment $M_{y, \text { max }}^{\mathrm{R}}(x=0)=M_{y}-l F_{z}$. In contrast to this, for parabolic and circular notch type hinges, the critical section is determined by the thinnest cross section $t_{s}$, as well, leading to a critical section very close to the thinnest cross section at $x \approx l / 2$, where the bending moment becomes $M_{y, \max }^{\mathrm{C}, \mathrm{P}}\left(x \approx \frac{l}{2}\right)=M_{y}-\frac{l}{2} F_{z}$. Thus, the maximum equivalent stresses are

$$
\begin{aligned}
& \sigma_{V, \text { max }}^{\mathrm{R}}=\sqrt{\left(\frac{F_{x}}{b t_{s}} K_{t x}^{\mathrm{R}}+\frac{6\left(M_{y}-l F_{z}\right)}{b t_{s}^{2}} K_{t b}^{\mathrm{R}}\right)^{2}+3\left(\frac{F_{z}}{2 b t_{s}}\right)^{2}}, \\
& \sigma_{V, \text { max }}^{\mathrm{C}, \mathrm{P}}=\sqrt{\left(\frac{F_{x}}{b t_{s}} K_{t x}^{\mathrm{C}, \mathrm{P}}+\frac{6\left(M_{y}-\frac{l}{2} F_{z}\right)}{b t_{s}^{2}} K_{t b}^{\mathrm{C}, \mathrm{P}}\right)^{2}+3\left(\frac{F_{z}}{2 b t_{s}}\right)^{2}} .
\end{aligned}
$$

The stress concentration factors for rectangular leaf type hinges $K_{t x}^{\mathrm{R}}, K_{t b}^{\mathrm{R}}$ strongly depend on the corner radius and can be found in Pilkey and Pilkey (2008). For circular and parabolic hinges, stress concentration factors can be approximated following Haibach (2006) 


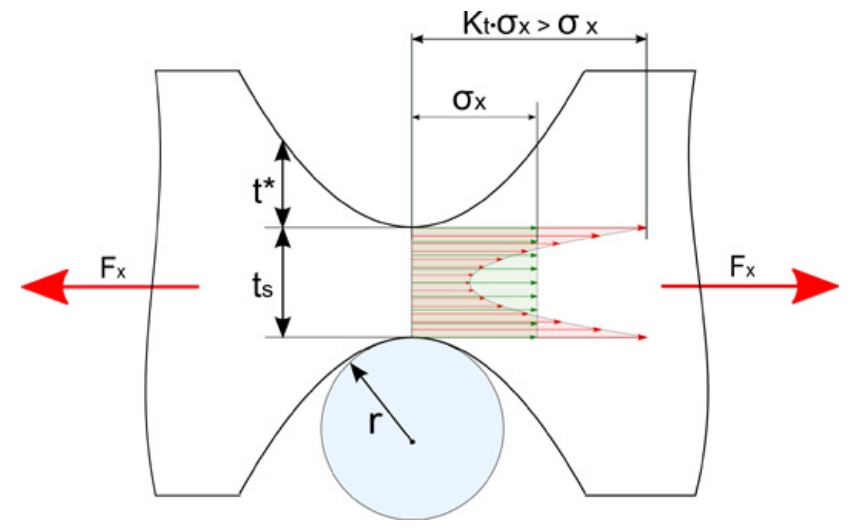

Figure 3. Stress concentration at flexure hinges due to notch effect.

$$
\begin{aligned}
K_{t x}^{\mathrm{C}, \mathrm{P}}=1+[ & 0.1\left(\frac{r}{t^{*}}\right)+0.7\left(1+\frac{t_{s}}{2 r}\right)^{2}\left(\frac{t_{s}}{2 r}\right)^{-3} \\
& \left.+0.13\left(\frac{t_{s}}{2 r}\right)\left(\frac{t_{s}}{2 r}+\frac{t^{*}}{r}\right)^{-1}\left(\frac{t^{*}}{r}\right)^{-1.25}\right]^{-\frac{1}{2}}, \\
K_{t b}^{\mathrm{C}, \mathrm{P}}=1+[ & 0.08\left(\frac{r}{t^{*}}\right)^{0.66}+2.2\left(1+\frac{t_{s}}{2 r}\right)^{2.25}\left(\frac{t_{s}}{2 r}\right)^{-3.375} \\
+ & \left.0.2\left(\frac{t_{s}}{2 r}\right)\left(\frac{t_{s}}{2 r}+\frac{t^{*}}{r}\right)^{-1}\left(\frac{t}{r}\right)^{-1.33}\right]^{-\frac{1}{2}},
\end{aligned}
$$

where $t^{*}=\frac{H-t_{s}}{2}$ and the radii of curvature $r$ are

$r^{\mathrm{C}}=\frac{\left.l^{2}+\left(H-t_{s}\right)^{2}\right)}{4\left(H-t_{s}\right)}=$ const.

and

$r^{\mathrm{P}}\left(x=\frac{l}{2}\right)=\frac{l^{2}}{4\left(H-t_{s}\right)}$,

for circular and parabolic hinges as shown in Fig. 3. Here, the geometric properties given in Eq. (2) and corresponding derivatives $t^{\prime}(x), t^{\prime \prime}(x)$ were applied to calculate the radius of a parabola $r^{\mathrm{P}}(x)=\left|\frac{\left(1+t^{\prime}\left(x^{2}\right)^{3 / 2}\right.}{t^{\prime \prime}(x)}\right|$.

Finally, the equivalent stresses can be calculated using Eq. (9) for known (nodal) loads and all considered types of flexure hinges considered in this work.

As an example, Table 2 illustrates the maximum elastic deformation $w(l)$ of differently-sized parabolic and circular flexure hinges based on given geometric parameter: length $l$, height $H=10 \mathrm{~mm}$, depth $b=10 \mathrm{~mm}$ and smallest thickness $t_{s}$. It can be noted, that circular flexure hinges provide a larger deflection range than parabolic counterparts maintaining the aforementioned common material points $P_{1}, P_{2}, P_{3}$.
Table 2. Maximum elastic deformation of circular (C) and parabolic $(\mathrm{P})$ flexure hinges undergoing bending due to pure shear force $F_{z}$.

\begin{tabular}{cccccccc}
\hline $\begin{array}{c}\text { Shape } \\
{[-]}\end{array}$ & $\begin{array}{c}l \\
{[\mathrm{~mm}]}\end{array}$ & $\begin{array}{c}t_{s} \\
{[\mathrm{~mm}]}\end{array}$ & $\begin{array}{c}K_{t x} \\
{[-]}\end{array}$ & $\begin{array}{c}K_{t b} \\
{[-]}\end{array}$ & $\begin{array}{c}F_{z} \\
{[\mathrm{~N}]}\end{array}$ & $\begin{array}{c}w(l) \\
{[\mu \mathrm{m}]}\end{array}$ & $\begin{array}{c}w^{\prime}(l) \\
{[\mathrm{rad}]}\end{array}$ \\
\hline $\mathrm{C}$ & 8 & 2 & 1.119 & 1.051 & 47.6 & 52.0 & -0.013 \\
$\mathrm{P}$ & 8 & 2 & 1.281 & 1.133 & 44.1 & 36.1 & -0.009 \\
\hline $\mathrm{C}$ & 9 & 1 & 1.040 & 1.015 & 12.2 & 93.2 & -0.020 \\
$\mathrm{P}$ & 9 & 1 & 1.102 & 1.043 & 11.8 & 65.8 & -0.015 \\
\hline $\mathrm{C}$ & 9.5 & 0.5 & 1.014 & 1.004 & 3.1 & 145.9 & -0.031 \\
$\mathrm{P}$ & 9.5 & 0.5 & 1.037 & 1.013 & 3.0 & 103.4 & -0.022 \\
\hline
\end{tabular}

Generally, the occurring stresses depend directly on the radii of curvature, i.e. smaller radii of curvature result in higher stress concentration factors leading to higher stresses. This is not a surprising result, however Eqs. (9)-(13) provide the reader with analytical expressions to calculate the range of elastic deformation of flexure hinges in compliant mechanisms prior to any modeling or manufacturing efforts.

\subsection{Stiffness and bending stiffness}

The stiffness $k_{x}, k_{z}$ and, in particular, the bending stiffness $c_{\psi}$ of a flexure hinge is important for modeling of compliant mechanisms using discrete spring joints or reduced finite element models. They are calculated analytically and compared to experimental data for all flexure hinge types considered in this work. The loads $\boldsymbol{F}=\left(F_{z}, M_{y}, F_{x}\right)^{T}$ and displacements $\boldsymbol{u}=(w(x), \psi(x), u(x, z))^{T}$ are coupled by the compliance expressions $\boldsymbol{u}=\mathbf{N} \boldsymbol{F}$ as given in Eq. (8). For modeling and topology optimization purposes, it is beneficial to convert this relation to

$\mathbf{K} u=F$

The stiffness matrix $\mathbf{K}$ represents all mechanical properties (for quasi-static problems) that are crucial for modeling purposes, topology optimization problems and (embedded) finite element calculations using efficient, reduced models.

Generally, these expressions are quite large, especially, for parabolic and circular flexure hinges. Due to conciseness, the stiffness matrix of a rectangular flexure hinge is presented solely. However, the calculation of stiffness matrices for parabolic and circular hinges is similar and straightforward. Using the derived relations between loads and corresponding displacement from Eq. (8) yield

$\mathbf{K}=E b t_{s}^{3}\left(\begin{array}{ccc}\frac{5}{l\left(12(1+v) t_{s}^{2}+5 l^{2}\right)} & \frac{5}{24(1+v) t_{s}^{2}+10 l^{2}} & 0 \\ \frac{5 l}{2 l\left(12(1+v) t_{s}^{2}+5 l^{2}\right)} & \frac{10 l^{2}+6(1+v) t_{s}^{2}}{l\left(12(1+v) t_{s}^{2}+5 l^{2}\right)} & 0 \\ 0 & 0 & \frac{1}{l l_{s}^{2}}\end{array}\right)$.

To compare these analytical calculations with experimental data with superposed external loads, scalar values 
Table 3. Bending stiffness $c_{\psi}$ for rectangular (R), circular (C) and parabolic $(\mathrm{P})$ flexure hinges: analytical calculations and experimental results.

\begin{tabular}{|c|c|c|c|c|c|}
\hline $\begin{array}{c}\text { Shape } \\
{[-]}\end{array}$ & $\begin{array}{c}1 \\
{[\mathrm{~mm}]}\end{array}$ & $\begin{array}{c}t_{s} \\
{[\mathrm{~mm}]}\end{array}$ & $\begin{array}{l}c_{\psi, \text { ana }} \\
{\left[\frac{\mathrm{Nm}}{\mathrm{rad}}\right]}\end{array}$ & $\begin{array}{c}c_{\psi, \exp } \\
{\left[\frac{\mathrm{Nm}}{\mathrm{rad}}\right]}\end{array}$ & $\begin{array}{r}\text { error } \\
\text { [\%] }\end{array}$ \\
\hline $\mathrm{P}$ & 8 & 2 & 199.37 & 180.06 & 10.72 \\
\hline $\mathrm{C}$ & 8 & 2 & 143.64 & 133.71 & 7.43 \\
\hline $\mathrm{R}$ & 8 & 2 & 58.33 & 61.18 & -4.66 \\
\hline
\end{tabular}

for bending stiffness $c_{\psi}$ are desirable. Therefore, the stiffness matrix is decomposed (diagonalized) into $\mathbf{K}_{\mathbf{D}}=$ $\operatorname{diag}\left(\lambda_{1}, \lambda_{2}, \lambda_{3}\right)$, where $\lambda_{1}, \lambda_{2}, \lambda_{3}$ are the eigenvalues of $\mathbf{K}$. The resulting eigenvectors $\mathbf{b}_{\mathbf{1}}, \mathbf{b}_{\mathbf{2}}, \mathbf{b}_{\mathbf{3}}$ are used to form the orthogonal transformation matrix $\mathbf{T}=\mathbf{T}\left(\mathbf{b}_{1}, \mathbf{b}_{2}, \mathbf{b}_{3}\right)$ which is negligibly close to identity $\mathbf{I}\left(\operatorname{norm}(\mathbf{T}-\mathbf{I}) \leq 10^{-4}\right)$ for every case considered in this work. Thus, Eq. (14) can be rewritten as

$\underbrace{\left(\begin{array}{ccc}\lambda_{1}=k_{z} & 0 & 0 \\ 0 & \lambda_{2}=c_{\psi} & 0 \\ 0 & 0 & \lambda_{3}=k_{x}\end{array}\right)}_{\mathbf{K}_{\mathbf{D}}} \underbrace{\left(\begin{array}{c}w \\ \psi \\ u\end{array}\right)}_{\boldsymbol{u}} \approx \underbrace{\left(\begin{array}{c}F_{z} \\ M_{y} \\ F_{x}\end{array}\right)}_{\boldsymbol{F}}$

yielding a desired, decoupled relation among loads, stiffness and deflection.

The bending stiffness $c_{\psi}$ for rectangular (R), circular (C) and parabolic $(\mathrm{P})$ flexure hinges are listed in Table 3. Here, the analytical calculations differ from the experimental results by a maximum relative error $\leq 11 \%$, which is acceptable, considering manufacturing imperfections in $\mathrm{z}$-direction and its enormous effect on the stiffness as described in detail in Ryu and Gweon (1997). Therefore, the aforementioned analytical expressions represent a good prediction for superposed, application-oriented loading conditions.

\subsection{Center of rotation}

The center of rotation and its motion with deflection of bodies connected by flexure hinges are crucial for a correct modeling of compliant mechanisms. Ignoring the particular center of rotation of flexure hinges can lead to parasitic motion or failure of the entire mechanism due to unwanted behavior, e.g. snap through effects.

The center of rotation is usually considered for rigid-bodymotions. However, many parts of a compliant mechanism that are connected by flexure hinges are very stiff and can be treated in a similar way. In this work, overall center of rotation $\mathbf{P}_{\mathrm{eff}}^{01}$ refers to a fixed point considering undeformed (0) and maximum elastically deformed state (1) as illustrated in Fig. 4, whereas its motion refers to the herpolhode, i.e. motion of instantaneous center of rotation with deflection.

In order to calculate the center of rotation of a rigid body attached to the flexure hinge, the position of two single points

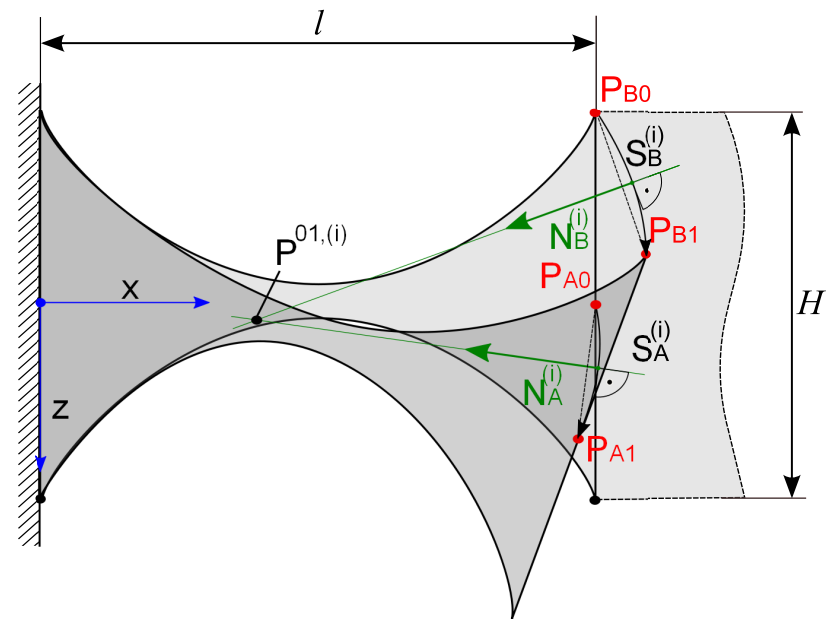

Figure 4. Determination of instantaneous center of rotation $\mathrm{P}^{01,(i)}$ of a flexure hinge.

$P_{A}, P_{B}$ and the corresponding displacements $u_{A}=u(l, 0)$, $w_{A}=w(l, 0)$ and $u_{B}=u(l,-H / 2)$ are considered. This yields an overall center of rotation

$\mathbf{P}_{\mathrm{eff}}^{01}=\frac{1}{2}\left(\begin{array}{c}\frac{w_{A}}{u_{A}-u_{B}} H+u_{A}+u_{B}+2 l \\ \frac{u_{A}}{u_{B}-u_{A}} H-\frac{u_{A} u_{B}}{w_{A}}+w_{A}\end{array}\right)$,

which holds for all types of flexure hinges.

The motion of the (instantaneous) center of rotation can be calculated considering an infinite number of intermediate steps between undeformed and maximum elastically deformed step. Therefore, the motion paths:

$\boldsymbol{S}_{A}^{(i)}=\boldsymbol{P}_{A}+\left(\begin{array}{c}u_{A}^{(i)} \\ w_{A}^{(i)}\end{array}\right), \quad \boldsymbol{S}_{B}^{(i)}=\boldsymbol{P}_{B}+\left(\begin{array}{c}u_{B}^{(i)} \\ w_{B}^{(i)}\end{array}\right)$,

the tangent vectors:

$\boldsymbol{T}_{A}^{(i)}=\dot{\boldsymbol{K}}_{\boldsymbol{A}}=\left(\begin{array}{c}\dot{u}_{A}^{(i)} \\ \dot{w}_{A}^{(i)}\end{array}\right), \quad \boldsymbol{T}_{B}^{(i)}=\dot{\boldsymbol{K}}_{\boldsymbol{B}}=\left(\begin{array}{c}\dot{u}_{B}^{(i)} \\ \dot{w}_{B}^{(i)}\end{array}\right)$,

and the corresponding normal vectors:

$\boldsymbol{N}_{A}^{(i)}=\left(\begin{array}{c}\dot{w}_{A}^{(i)} \\ -\dot{u}_{A}^{(i)}\end{array}\right), \quad \boldsymbol{N}_{B}^{(i)}=\left(\begin{array}{c}\dot{w}_{B}^{(i)} \\ -\dot{u}_{B}^{(i)}\end{array}\right)$,

need to be calculated first. Determining the point of intersection of $N_{A}^{(i)}$ and $N_{B}^{(i)}$ leads to the center of rotation for all intermediate steps, i.e. the desired motion with deflection:

$\mathbf{P}^{01,(i)}=\left(\begin{array}{l}\frac{2\left(u_{A}+l\right) \dot{u}_{A}-2\left(u_{B}+l\right) \dot{u}_{B}+H \dot{w}_{A}}{2\left(\dot{u}_{A}-\dot{u}_{B}\right)} \\ w_{A}-\frac{2\left(u_{A}-u_{B}\right) \dot{u}_{B}+H \dot{w}_{A}}{2\left(\dot{u}_{A}-\dot{u}_{B}\right) \dot{w}_{A}} \dot{u}_{A}\end{array}\right)$, 
Table 4. Overall center of rotation for rectangular $(\mathrm{R})$, circular $(\mathrm{C})$ and parabolic $(\mathrm{P})$ flexure hinges.

\begin{tabular}{ccccccc}
\hline $\begin{array}{c}\text { Shape } \\
{[-]}\end{array}$ & $\begin{array}{c}1 \\
{[\mathrm{~mm}]}\end{array}$ & $\begin{array}{c}t_{s} \\
{[\mathrm{~mm}]}\end{array}$ & $\begin{array}{c}F_{z} \\
{[\mathrm{~N}]}\end{array}$ & $\begin{array}{c}M_{y} \\
{[\mathrm{Nm}]}\end{array}$ & $\begin{array}{c}\mathrm{P}_{\mathrm{eff}, \Delta x}^{01} \\
{[\mathrm{~mm}]}\end{array}$ & $\begin{array}{c}\mathrm{P}_{\mathrm{eff}, z}^{01} \\
{[\mathrm{~mm}]}\end{array}$ \\
\hline $\mathrm{R}$ & 8 & 2 & 35 & -1.260 & -0.1479 & 0.0499 \\
$\mathrm{C}$ & 8 & 2 & 35 & -1.260 & -0.0657 & 0.0191 \\
$\mathrm{P}$ & 8 & 2 & 35 & -1.260 & -0.0615 & 0.0143 \\
\hline $\mathrm{R}$ & 9 & 1 & 8 & -0.288 & -0.1607 & 0.1168 \\
$\mathrm{C}$ & 9 & 1 & 8 & -0.288 & -0.0142 & 0.0307 \\
$\mathrm{P}$ & 9 & 1 & 8 & -0.288 & -0.0070 & 0.0222 \\
\hline
\end{tabular}

The effective centers of rotation for rectangular (R), circular $(\mathrm{C})$ and parabolic $(\mathrm{P})$ flexure hinges are illustrated in Table 4 for two different loading conditions. Here, the $x$ coordinates refer to the center of the flexure hinge denoted by $\Delta x$. It can be noted, that the effective center of rotation is shifted to $(-x,+z)$ direction for all flexure hinges for the given load case. The motion of the center of rotation for rectangular flexure hinges is clearly larger due to the deflection of the entire hinge length $l$, whereas it is very small for circular and parabolic flexure hinges. Thus, it is crucial to consider the center of rotation and its motion with deflection for rectangular flexure hinges in order to ensure an appropriate modeling.

\subsection{Natural frequency}

The natural frequency of a system consisting of a rigid body connected to a flexure hinge as shown in Fig. 5 (left) is relevant for compliant mechanisms under dynamic loading conditions. In addition, it is a good quality measure comparing analytical, numerical and experimental studies.

The natural frequencies are first calculated analytically using the aforementioned Timoshenko's beam theory. Extending Eq. (7) to a dynamic state yield the differential equations:

$$
\begin{aligned}
\rho A(x) \ddot{w}(x)-\left(G A_{*}(x)\left(w^{\prime}(x)+\psi\right)\right)^{\prime} & =q, \\
\rho I_{y}(x) \ddot{\psi}-\left(E I_{y}(x) \psi^{\prime}\right)^{\prime}+G A_{*}\left(w^{\prime}+\psi\right) & =0 .
\end{aligned}
$$

Applying standard boundary conditions at fixed end $x=0$

$w=0$,

$\psi=0$,

and free end $x=l$

$$
\begin{aligned}
E I_{y} \psi^{\prime} & =0, \\
G A_{*}\left(w^{\prime}+\psi\right) & =0,
\end{aligned}
$$

the differential Eq. (22) can be solved. However, solving these equations analytically for a variable, unspecified thick-
Table 5. Natural frequencies for rectangular (R), circular $(\mathrm{C})$ and parabolic $(\mathrm{P})$ flexure hinges connected to a rigid body $(H=10 \mathrm{~mm}$, $L=50 \mathrm{~mm}, b=10 \mathrm{~mm}$ ).

\begin{tabular}{ccccccc}
\hline & $\begin{array}{c}1 \\
{[\mathrm{~mm}]}\end{array}$ & $\begin{array}{c}t_{s} \\
{[\mathrm{~mm}]}\end{array}$ & $\begin{array}{c}f_{\text {ana }} \\
{[\mathrm{Hz}]}\end{array}$ & $\begin{array}{c}f_{\text {num }} \\
{[\mathrm{Hz}]}\end{array}$ & $\begin{array}{c}f_{\exp } \\
{[\mathrm{Hz}]}\end{array}$ & $\begin{array}{c}f_{\exp }^{\mathrm{dm}} \\
{[\mathrm{Hz}]}\end{array}$ \\
\hline $\mathrm{R}$ & 9.47 & 1.18 & $146.2(7.3 \%)$ & $144.0(5.7 \%)$ & 136.2 & $141.0(3.9 \%)$ \\
$\mathrm{C}$ & 9.09 & 0.99 & $218.6(8.3 \%)$ & $219.7(7.8 \%)$ & 238.4 & $242.5(1.7 \%)$ \\
$\mathrm{P}$ & 9.09 & 1.07 & $284.8(0.2 \%)$ & $274.0(4.0 \%)$ & 285.3 & $288.5(1.1 \%)$ \\
\hline $\mathrm{R}$ & 8.42 & 2.28 & $408.4(10.9 \%)$ & $400.9(8.9 \%)$ & 368.1 & $370.0(0.9 \%)$ \\
$\mathrm{C}$ & 8.08 & 2.00 & $534.9(2.9 \%)$ & $524.4(4.8 \%)$ & 550.9 & $541.6(1.7 \%)$ \\
$\mathrm{P}$ & 8.03 & 2.13 & $676.9(6.0 \%)$ & $643.3(0.8 \%)$ & 638.4 & $636.4(0.3 \%)$ \\
\hline
\end{tabular}
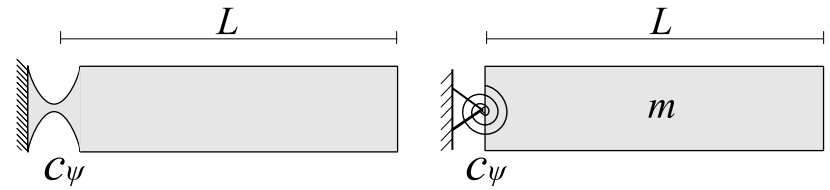

Figure 5. Continuous flexure hinge (left) and equivalent discrete torsion spring model (right).

ness $t(x) \neq$ const. is not always possible. Therefore, a numerical approach, namely the Rayleigh quotient

$\omega_{1}^{2}=\frac{\max E_{\mathrm{p}}}{\max E_{k}}$

is chosen, where the first natural circular frequency $\omega_{1}$ is approximated by the ratio of maximum values of potential and kinetic energies $E_{\mathrm{p}}$ and $E_{\mathrm{k}}$. Following Tabarrok and Karnopp (1967) yield

$\omega_{1}^{2}=\frac{\int_{0}^{l} E I_{y}(x) \Psi^{\prime}(x)^{2}+G A_{*}(x)\left(W^{\prime}(x)+\Psi(x)\right)^{2} d x}{\rho \int_{0}^{l} A(x) W(x)^{2}+I_{y}(x) \Psi(x)^{2} d x}$,

where displacement and bending angle are described by appropriate test functions $\Psi(x)$ and $W(x)$. In order to determine $\Psi(x)$ and $W(x)$, the displacements and bending angle occuring from a uniform transverse load $q_{0}$ with a resulting bending moment $M_{y}(x)=-\frac{1}{2} q_{0}(l-x)^{2}$, as suggested in Rao (2007), are used.

Table 5 lists the analytically, numerically and experimentally determined first natural frequencies $f_{\text {ana }}, f_{\text {num }}, f_{\exp }, f_{\exp }^{\mathrm{dm}}$ for different flexure hinges connected to a rigid body, as shown in Fig. 5 (left). The analytical calculations $f_{\text {ana }}$ are based on Eq. (26) using Timoshenko's beam theory. The numerical calculations $f_{\text {num }}$ are obtained by a numerical modal analysis using the commercial software package Abaqus 6.9. The experimental data $f_{\exp }$ is gathered by a experimental modal analysis using non-contact laser scanning vibrometer system. The frequencies $f_{\exp }^{\mathrm{dm}}$ are calculated using the standard relation

$f_{\exp }^{\mathrm{dm}}=\frac{1}{2 \pi} \sqrt{\frac{c_{\psi, \exp }}{I_{\mathrm{m}}}}$, 
with mass inertia $I_{\mathrm{m}}$, that holds for discrete models of a torsional (bending vibration, as illustrated in Fig. 5 (right).

It can be noted, that the analytical calculation agrees well with the numerical results on all types of flexure hinges. Compared to the experimental data, a relative error less than $11 \%$ can be noted. Due to imperfections in the manufacturing process of the specimen, this error seems acceptable to the authors; cf. Ryu and Gweon (1997). Comparing the experimental data $f_{\exp }$ and $f_{\exp }^{\mathrm{dm}}$ with each other, a very small relative error of less than $4 \%$ can be noted. This implies, that flexure hinges can be modeled by discrete torsional springs as illustrated in Fig. 5, using the bending stiffness $c_{\psi}$ calculated in Sect. 3.5.

\section{Conclusion: benefits for the synthesis of compliant mechanisms}

In this work, planar flexure hinges are investigated in terms of their application in the synthesis of compliant mechanisms, where one-node hinges occur as an artificial artefact of many topology optimization methods. In order to replace these pseudo-hinges by flexure hinges efficiently, a characterization of different types of flexure hinges was done.

Relevant mechanical properties, such as displacement and bending angle, mechanical stresses, bending stiffness, center of rotation, maximum elastic deformation and first natural frequencies were derived analytically and agree well with numerical and experimental data.

The analytical expressions were derived based on Timoshenko's beam theory taking into account shear deformation of flexure hinges. In order to calculate an elastic deflection range, von-Mises yield criterion was chosen. Numerical simulations were performed using commercial software package Abaqus 6.9. Experimental results of bending stiffness and natural frequencies were gathered using a tension test machine and a non-contact scanning laser vibrometer system.

More importance was given to practice-oriented flexure hinge types in terms of cost-saving manufacturability, i.e. circular notch type hinges and rectangular leaf type hinges, as well as well-customizable parabolic hinges. Comparing different types of flexure hinges of similar dimensions, the following conclusion can be drawn:

Rectangular geometry of flexure hinges yield low bending stiffness and very high rotational deflection, while the location of the center of rotation and its motion with deflection needs to be taken into account.

Circular geometry of flexure hinges yields moderate bending stiffness and high rotational deflection, while the center of rotation remains close to the center point of the hinge.

Parabolic geometry of flexure hinges yield high bending stiffness and low rotational deflection, while the center of rotation remains very close to the center point of the hinge.
Some of these conclusions are not surprising, however the key results of this work are the analytical expressions that enable the reader:

- to calculate the relevant mechanical properties of flexure hinges explicitly and

- to select the appropriate type of flexure hinge based on the (known) nodal loads and displacements resulting from the synthesis of compliant mechanism,

prior to any modeling or manufacturing efforts. Thus, the synthesis and manufacturing process of compliant mechanisms can be accelerated.

Acknowledgements. This research was supported by priority programme grant SPP1476 from the German Research Foundation (DFG) to both authors, and by a doctoral research stipend from the German Academic Exchange Service (DAAD-D/10/46834) to the first author. In addition, the authors would like to thank several students, in particular M. Anselmann, for their studies on flexure hinges.

Edited by: N. Tolou

Reviewed by: two anonymous referees

\section{References}

Ananthasuresh, G. and Kota, S.: Designing compliant mechanisms, Mech. Eng., 117, 93-96, 1995.

Ansola, R., Canales, J., Tárrago, J., and Rasmussen, J.: An integrated approach for shape and topology optimization of shell structures, Comput. Struct., 80, 449-458, 2002.

Bendsøe, M. and Sigmund, O.: Topology optimization: theory, methods, and applications, Springer Verlag, 2003.

Bendsøe, M. and Sigmund, O.: Optimization of structural topology, shape, and materials, ORBIT, 2008.

Bruns, T. and Tortorelli, D.: Topology optimization of non-linear elastic structures and compliant mechanisms, Comput. Method Appl. M., 190, 3443-3459, 2001.

Conway, H. and Nickola, W.: Anticlastic action of flat sheets in bending, Exp. Mech., 5, 115-119, 1965.

Frecker, M. I., Ananthasuresh, G. K., Nishiwaki, S., Kikuchi, N., and Kota, S.: Topological Synthesis of Compliant Mechanisms Using Multi-Criteria Optimization, J. Mech. Design, 119, 238 245, 1997.

Haibach, E.: Betriebsfestigkeit: Verfahren und Daten zur Bauteilberechnung, Springer New York, 2006.

Howell, L.: Compliant mechanisms, Wiley-Interscience, 2001.

Lobontiu, N.: Compliant mechanisms: design of flexure hinges, CRC, 2003.

Love, A.: A treatise on the mathematical theory of elasticity, at the University Press, 1920.

Mattson, C., Howell, L., and Magleby, S.: Development of commercially viable compliant mechanisms using the pseudo-rigidbody model: Case studies of parallel mechanisms, J. Intel. Mat. Syst. Str., 15, 195-202, 2004. 
Paros, J. and Weisbord, L.: How to design flexural hinges, Mach. Des., 25, 151-156, 1965.

Pilkey, W. D. and Pilkey, D. F.: Peterson's stress concentration factors, John Wiley \& Sons, 2008.

Poulsen, T.: A simple scheme to prevent checkerboard patterns and one-node connected hinges in topology optimization, Struct. Multidiscip. O., 24, 396-399, 2002.

Raatz, A.: Stoffschlüssige Gelenke aus pseudo-elastischen Formgedächtnislegierungen in Pararellrobotern, Vulkan-Verlag $\mathrm{GmbH}, 2006$.

Rao, S.: Vibration of continuous systems, John Wiley \& Sons Inc, 2007.

Ryu, J. and Gweon, D.: Error analysis of a flexure hinge mechanism induced by machining imperfection, Precis. Eng., 21, 8389, 1997.
Saxena, A. and Ananthasuresh, G.: On an optimal property of compliant topologies, Struct. Multidiscip. O., 19, 36-49, 2000.

Sigmund, O.: Manufacturing tolerant topology optimization, Acta Mech. Sinica, 25, 227-239, 2009.

Smith, S.: Flexures: elements of elastic mechanisms, CRC, 2000.

Tabarrok, B. and Karnopp, B.: Analysis of the oscillations of the Timoshenko beam, Z. Angew. Math. Phys., 18, 580-587, 1967.

Wang, C., Reddy, J., and Lee, K.: Shear deformable beams and plates, Elsevier, Amsterdam, 2000.

Yoon, G., Kim, Y., Bendsøe, M., and Sigmund, O.: Hinge-free topology optimization with embedded translation-invariant differentiable wavelet shrinkage, Struct. Multidiscip. O., 27, 139150, 2004. 